\title{
Effect of cell membrane structure of human erythrocyte on its electrophoresis
}

\author{
Jyh-Ping $\mathrm{Hsu}^{\mathrm{a}, *}$, Sung-Hwa Lin ${ }^{\mathrm{a}}$, Shiojenn Tseng ${ }^{\mathrm{b}}$ \\ a Department of Chemical Engineering, National Taiwan University, Taipei 10617, Taiwan, ROC \\ ${ }^{\mathrm{b}}$ Department of Mathematics, Tamkang University, Tamsui, Taipei 25137, Taiwan, ROC
}

Received 18 April 2003; received in revised form 23 June 2003; accepted 23 June 2003

\begin{abstract}
The electrophoretic behavior of human erythrocyte is investigated theoretically. The present analysis extends previous studies to that the effect of the membrane structure of a cell is taken into account. The result obtained provides a useful tool for the estimation of the surface properties of human erythrocyte through electrophoretic measurements. Fitting the present model to the experimental data of Haydon and Seaman [Arch. Biochem. Biophys. 122 (1967) 126], for example, reveals that the surface charge of erythrocyte arises mainly from the dissociation of three functional groups, which bond to different sites on cell membrane : (a) $N$-acetylneuraminic acid (sialic acid) with $\mathrm{p}_{\mathrm{HA}_{\mathrm{Naa}}}=2.6$, (b) some kind of $\alpha$-carboxylic acid with $\mathrm{p} K_{\mathrm{HA}_{\alpha-\mathrm{c}}}=3.35$, (c) weak amino base functional group with $\mathrm{p} K_{\mathrm{BH}^{+}}=8.5$. The distribution of $\mathrm{Na}^{+}$in the protein layer of a cell is found to vary with the $\mathrm{pH}$ of the bulk liquid phase, the possible implication of this phenomenon to cell physiology is discussed.
\end{abstract}

(C) 2003 Elsevier B.V. All rights reserved.

Keywords: Cell membrane structure; Human erythrocyte; Electrophoresis; Mathematical model

\section{Introduction}

Erythrocyte is one of the earliest and extensively analyzed blood cells in blood physiological and clinical studies. Detailed information about the constituents of human erythrocyte and their associated functional mechanisms is now available. This includes, for example, the fundamental structure of cell, the quantities of nutrients in each part

\footnotetext{
* Corresponding author. Tel.: +886-2-2363-7448; fax: + 886-2-2362-3040.

E-mail address: jphsu@ccms.ntu.edu.tw (J.-P. Hsu).
}

of cell, the way each metabolic cycle is working and the connections between them, and the architecture and functions of chromosomes, to name a few. In fact, the locating and mechanism of replications for genes of human red blood cell are almost complete in the past decade. Despite of this, topics relevant to erythrocyte such as its intracellular and extracellular proteins still draw the attention of numerous researchers. Often, using advanced instruments is necessary, and the experimental works involve laborious and tedious procedures. However, reaching conclusions that are controversial is not uncommon in practice. 
Electrophoresis is one of the basic yet powerful analytical tools for biological studies. Several essential properties of cells and protein macromolecules such as the quantity of their compositions and charged conditions can be estimated without too much difficulty through electrophoretic measurements. Analysis about electrophoretic phenomenon was pioneered by Smoluchowski [1]. Based on the assumptions of creeping flow, dilute dispersion, and thin double layer, he was able to derive the following expression for the electrophoretic mobility of a rigid entity $\mu_{e}$ :

$\mu_{e}=\frac{\varepsilon_{s} \zeta}{\eta}$

where $\zeta$ is the zeta potential of the entity, and $\varepsilon_{s}$ and $\eta$ are, respectively, the permittivity and the viscosity of the liquid phase. In practice, $\zeta$ is often estimated based on an experimentally measured $\mu_{e}$. For the case of a non-rigid entity such as biological cell, which possesses an ion-penetrable protein layer near its surface, Eq. (1) needs to be modified accordingly. Several attempts have been made to take this factor into account [2-10]. It was found that the presence of an ion-penetrable layer on the surface of an entity has the effect of lowering its electrophoretic mobility, in general. In previous studies, the surface layer is assumed to have a uniform structure. While this assumption is satisfactory for entities covered by an artificial membrane, it can be unrealistic for biological cells. This is because the structure of the membrane of human erythrocyte is of complicated nature; it comprises about 49.2 wt.\% proteins, 43.6 wt.\% lipids, and nutrients such as glycoproteins, glycolipids, and carbohydrate materials, and others. The lipids are of amphipathic nature possessing a hydrophilic head at one end of the lipid chain, and a hydrophobic tail at the other end. When placed in an aqueous environment, the lipids will align themselves straightly to form a lipid bi-layer through arranging the hydrophobic tails inside and the hydrophilic heads facing to the aqueous side so that a stable state is reached. According to SEM observations and analytical measurements, erythrocyte membrane appears to have the structure shown in Fig. 1 [11], where it has a lipid bi- layer inlaid by many kinds of membrane proteins, which have outer cone-like externals. This sketch for the cell membrane is sometimes called the fluid-mosaic model, because the lipid bi-layer is of dynamic nature, which may move longitudinally and laterally sometimes depending on the packing degree of the lipids to each other. According to the state of their contact to lipids, membrane proteins can be classified into the surface membrane protein and the integral membrane protein; the later is also called transmembrane protein if it spans the entire width of the lipid bi-layer. The arrangements of lipids and proteins depend upon the hydrophilic, the hydrophobic, and the ionic forces between them and the body fluid.

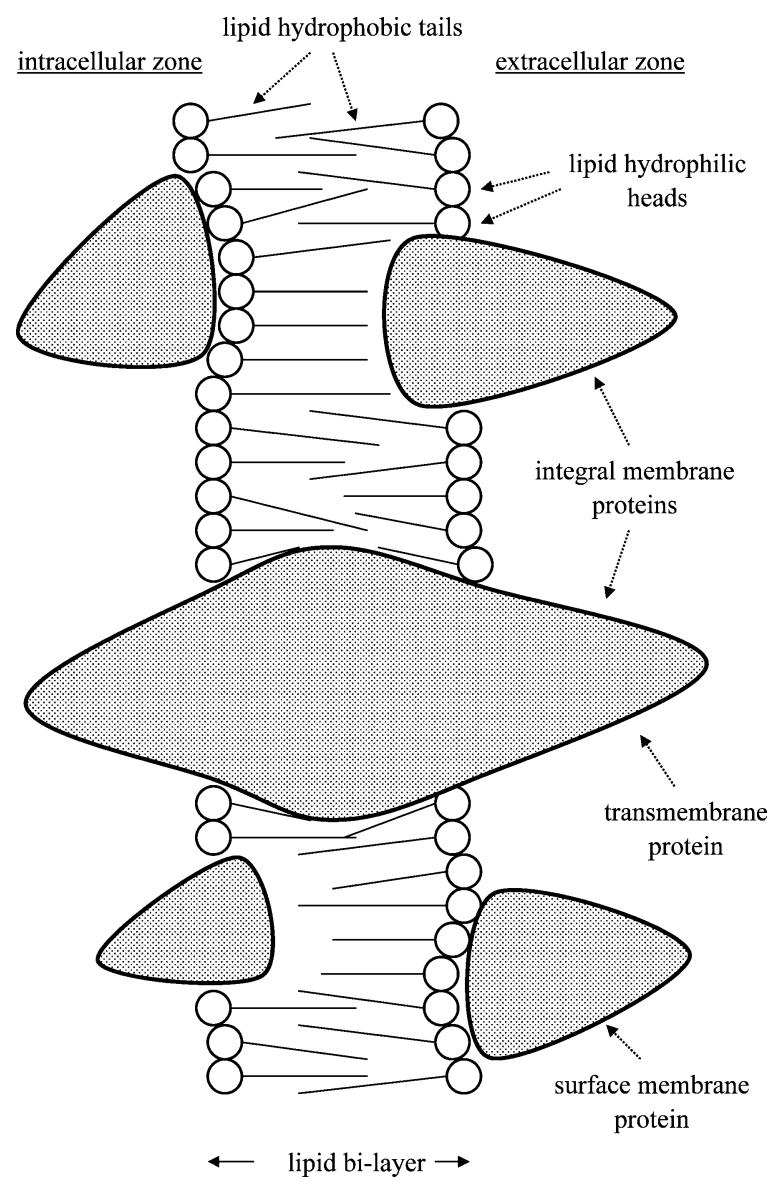

Fig. 1. Schematic representation of the structure of erythrocyte membrane. 
In a series studies on the surface properties of human red blood cells, Seaman and coworkers [12-18] conducted a considerable amount of electrophoretic experiments. Based on the classic Gouy-Chapman double layer model and a quantitatively chemical analysis $[15,16]$, it was verified that the charge on erythrocyte membrane arises mainly from the dissociations of the bonded ninecarbon sugar, $N$-acetylneuraminic acid $\left(\mathrm{p} K_{\mathrm{HA}_{\mathrm{Naa}}}=2.6\right)$, and some unknown membraneprotein-bound $\alpha$-carboxylic acid $\left(\mathrm{p} K_{\mathrm{HA}_{\alpha-\mathrm{ca}}}=\right.$ 3.35). The contributions of these functional groups to membrane charge are 61.5 and $38.5 \%$, respectively. However, some disagreement between their experimental data and the corresponding theoretical values was observed when the level of $\mathrm{pH}$ is high. It can be inferred that this disagreement is due to the fact that the effect of the dissociable and basic membrane-protein-bonded amino functional groups is neglected in their analysis, because if $\mathrm{pH}$ is high, their presence becomes significant. In the present analysis, the model of Haydon and Seaman [16] is modified to take this effect into account. In addition, the structure of the protein layer of cell membrane is considered to make the description of the electrophoretic behavior of a cell more realistic.

\section{Theory}

Human erythrocyte is disk-shaped with radius in the range $5-9 \times 10^{-6} \mathrm{~m}$ and thickness close to $10^{-6} \mathrm{~m}$ [11]. According to Smoluchowski [1], if the double layer surrounding an entity is thin, then its electrophoretic behavior is independent of its shape. Therefore, we consider the one-dimensional problem illustrated in Fig. 2 where the depth for the membrane protein extending into the liquid phase, $d_{p l}$, is defined as the thickness of the protein layer, and $d_{l l}$ be the thickness of lipid bi-layer. The magnitudes of $d_{l l}$ and $d_{p l}$ are about $75 \times 10^{-10}$ and $40-110 \times 10^{-10} \mathrm{~m}$, respectively [16]. For illustration, $d_{p l}$ is assigned the value $75 \times 10^{-10}$ $\mathrm{m}$ in subsequent discussion. For convenience, the Cartesian coordinates with its origin located on the lipid bi-layer/protein layer interface are adopted. The outward hydrophilic surface $(x=0)$

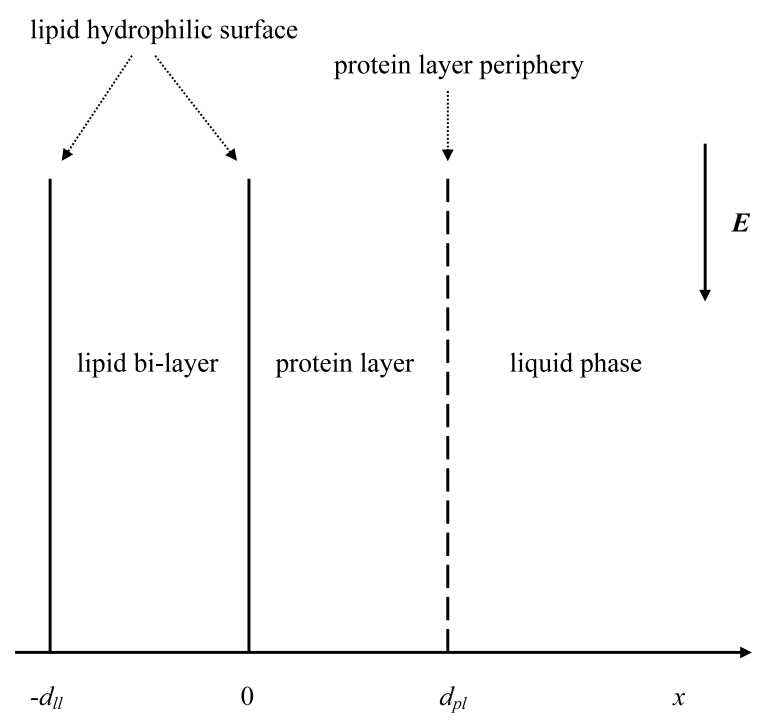

Fig. 2. Schematic representation of the problem considered. The origin of the Cartesian coordinates is located at the lipid bilayer/protein layer interface; $d_{l l}$ and $d_{p l}$ are, respectively, the thickness of lipid bi-layer and that of protein layer.

of the lipid bi-layer is assumed to be rigid to mobile ions, and the thickness of lipid bi-layer is fixed. Referring to Fig. 2, an electrical field $\boldsymbol{E}$ parallel to the surface of cell membrane with strength $E$ is applied. The liquid phase is a standard saline buffer solution prepared mainly by dilution of the following materials in water: sobitol, which acting as isotonic substance, $3 \times$ $10^{-4} \mathrm{M} \mathrm{Na} \mathrm{Na}_{3}$ buffer solution, and varied concentrations of $\mathrm{NaCl}$ for ionic strength adjustment. The $\mathrm{pH}$ of the saline solution is adjusted by $\mathrm{NaOH}$ and $\mathrm{HCl}$ solutions. Because the concentration of $\mathrm{Na}_{2} \mathrm{CO}_{3}$ is low, the condition of electroneutrality in the bulk liquid phase requires that:

$C_{\mathrm{Na}^{+}}^{b}+C_{\mathrm{H}^{+}}^{b}-C_{\mathrm{Cl}^{-}}^{b}-C_{\mathrm{OH}^{-}}^{b} \cong 0$

where $C_{\mathrm{Na}^{+}}{ }^{b}, C_{\mathrm{H}^{+}}{ }^{b}, C_{\mathrm{Cl}^{-}}{ }^{b}$, and $C_{\mathrm{OH}^{-}}{ }^{b}$ are, respectively, the bulk concentrations of $\mathrm{Na}^{+}$, $\mathrm{H}^{+}, \mathrm{Cl}^{-}$, and $\mathrm{OH}^{-}$. Suppose that the spatial variation of the concentration of ionic species follows Boltzmann distribution. Then based on the Gauss law, it can be shown that the electrical potential $\psi$ is described by the Poisson-Boltzmann equation: 


$$
\begin{aligned}
& \varepsilon_{s} \frac{d^{2} \psi}{d x^{2}}=-\rho_{e l} \\
&=-F\left[C_{\mathrm{Na}^{+}}^{b} \exp (-F \psi / R T)\right. \\
&+C_{\mathrm{H}^{+}}^{b} \exp (-F \psi / R T) \\
&-C_{\mathrm{Cl}^{-}}^{b} \exp (F \psi / R T) \\
&\left.\quad-C_{\mathrm{OH}^{-}}^{b} \exp (F \psi / R T)\right] \\
&= 2 I_{s} F \sinh (F \psi / R T), \\
& d_{p l} \leq x \\
& \frac{d}{d x}\left(\begin{array}{l}
\varepsilon_{p l} \\
d x
\end{array}\right)=-\rho_{e l}+F\left(C_{\mathrm{A}_{\mathrm{Naa}}^{-}}+C_{\mathrm{A}_{\alpha-\mathrm{ca}}^{-}}-C_{\mathrm{BH}^{+}}\right) \\
&=2 I_{s} F \sinh (F \psi / R T) \\
&+ F\left(C_{\mathrm{A}_{\mathrm{Naa}}^{-}}+C_{\mathrm{A}_{\alpha-\mathrm{ca}}^{-}}-C_{\mathrm{BH}^{+}}\right), \\
& 0 \leq x \leq d_{p l}
\end{aligned}
$$

In these expressions $F, R$, and $T$ are, respectively, the Faraday constant, the gas constant, and the absolute temperature, and $\varepsilon_{s}$ and $\varepsilon_{p l}$ are, respectively, the permittivity of the liquid phase and that of protein layer. Here, we assume that $\varepsilon_{s}$ can be approximated by the permittivity of water. $\rho_{e l}$ and $I_{s}$ are, respectively, the space charge density of mobile ions and the ionic strength of the liquid phase with $I_{s}=C_{\mathrm{Na}^{+}}{ }^{b}+C_{\mathrm{H}^{+}}{ }^{b}=C_{\mathrm{Cl}^{-}}{ }^{b}+C_{\mathrm{OH}^{-}}{ }^{b}$. $C_{\mathrm{A}_{\mathrm{Naa}}^{-}}, C_{\mathrm{A}_{\alpha-\mathrm{ca}}^{-}}$, and $C_{\mathrm{BH}^{+}}$denote, respectively, the concentrations of the ionized functional groups of $N$-acetylneuraminic acid, $\alpha$-carboxylic acid, and amino base in the protein layer. Note that because cells are usually fixed by treating with aldehydes in the experiment $[15,16]$, the charges arising from other cell-membrane-adsorbed polymeric ions such as hemoglobin, antibodies, and perhaps intracellular constituents, can be ignored. The dissociation of the predominant functional groups can be expressed by:

$$
\begin{aligned}
& \mathrm{HA}_{\mathrm{Naa}}=\mathrm{H}^{+}+\mathrm{A}_{\mathrm{Naa}}^{-}, \\
& K_{\mathrm{HA}_{\mathrm{Naa}}}=\frac{C_{\mathrm{H}^{+}} C_{\mathrm{A}_{\text {Naa }}^{-}}}{C_{\mathrm{HA}_{\mathrm{Naa}}}} \\
& \mathrm{HA}_{\alpha-\mathrm{ca}}=\mathrm{H}^{+}+\mathrm{A}_{\alpha-\mathrm{ca}}^{-}, \\
& K_{\mathrm{HA}_{\alpha-\mathrm{ca}}}=\frac{C_{\mathrm{H}^{+}} C_{\mathrm{A}_{\alpha-\mathrm{ca}}^{-}}}{C_{\mathrm{HA}_{\alpha-\mathrm{ca}}}}
\end{aligned}
$$

$\mathrm{BH}^{+}=\mathrm{H}^{+}+\mathrm{B}, \quad K_{\mathrm{BH}^{+}}=\frac{C_{\mathrm{H}^{+}} C_{\mathrm{B}}}{C_{\mathrm{BH}^{+}}}$

In these expression, $\mathrm{H}^{+}$and $C_{\mathrm{H}^{+}}$are, respectively, the hydrogen ion and its concentration. $\mathrm{HA}_{\mathrm{Naa}}$, $\mathrm{HA}_{\alpha-\text { ca }}$, and $\mathrm{B}$ are, respectively, the non-ionized functional groups of $N$-acetylneuraminic acid, $\alpha$ carboxylic acid, and amino base, $C_{\mathrm{HA}_{\mathrm{Naa}}}, C_{\mathrm{HA}_{\alpha-\mathrm{ca}}}$, and $C_{\mathrm{B}}$ are, respectively, the concentrations of these non-ionized functional groups, $\mathrm{A}_{\overline{\mathrm{Naa}}}, \mathrm{A}_{\alpha-\mathrm{ca}}^{-}$, and $\mathrm{BH}^{+}$are the ionized state of them, respectively, and $K_{\mathrm{HA}_{\mathrm{Naa}}}, K_{\mathrm{HA}_{\alpha-\mathrm{ca}}}$, and $K_{\mathrm{BH}^{+}}$are the corresponding dissociation constants. On the basis of Eqs. (4a), (4b) and (4c) and the conservation of each species, it can be shown that:

$$
\begin{aligned}
C_{\mathrm{A}_{\mathrm{Naa}}^{-}} & =\frac{C_{\mathrm{HA}_{\mathrm{Naa}}}^{0}}{1+C_{\mathrm{H}^{+}}^{b} \exp (-F \psi / R T) / K_{\mathrm{HA}_{\mathrm{Naa}}}} \\
C_{\mathrm{A}_{\alpha-\mathrm{ca}}^{-}} & =\frac{C_{\mathrm{HA}_{\alpha-\mathrm{ca}}}^{0}}{1+C_{\mathrm{H}^{+}}^{b} \exp (-F \psi / R T) / K_{\mathrm{HA}_{\alpha-\mathrm{ca}}}} \\
C_{\mathrm{BH}^{+}} & =\frac{C_{\mathrm{B}}^{0}}{1+K_{\mathrm{BH}^{+}} / C_{\mathrm{H}^{+}}^{b} \exp (-F \psi / R T)}
\end{aligned}
$$

where $C_{\mathrm{HA}_{\mathrm{Naa}}}{ }^{0}, C_{\mathrm{HA}_{\alpha-\mathrm{ca}}}{ }^{0}$, and $C_{\mathrm{B}}^{0}$ denote, respectively, the total concentrations of $\mathrm{HA}_{\mathrm{Naa}}, \mathrm{HA}_{\alpha-\mathrm{ca}}$, and $\mathrm{B}$. The precise locations of these functional groups on cell membrane are not known, and we assume that they are distributed randomly and $C_{\mathrm{HA}_{\mathrm{Naa}}}{ }^{0}, C_{\mathrm{HA}_{\alpha-\mathrm{ca}}}{ }^{0}$, and $C_{\mathrm{B}}^{0}$ are constant. Usually, protein chains are randomly distributed in the protein layer and have varied dimensions. However, SEM observations revealed that proteins on cell membrane appear in the form of cone-like externals protruding from the lipid bi-layer [11]. Because they are immersed in the aqueous solution, the spatial distribution of the outer parts of these proteins may be Gaussian, just as that of a polymer chain in a dilute medium. However, because the parameters of the corresponding Gauss distribution are not available at the present stage, we assume that the outer part of a protein chain is cone-shaped with bottom diameter $2 d_{p l}$ for simplicity. Therefore, the spatial variation of the protein units, i.e. amino acid segments, in the $x$-direction can be described by: 
$n_{m}=3 \bar{n}_{m}\left(1-\frac{x}{d_{p l}}\right)^{2}$

where $n_{m}$ is the number of protein units per unit volume of protein layer, and $\bar{n}_{m}$ is its average value. Since the permittivity of protein layer is directly related to the space density of the protein units inside, the spatial variation of $\varepsilon_{p l}$ can be described by:

$\varepsilon_{p l}=\varepsilon_{s}-\frac{4}{3} \pi \bar{a}_{m}^{3}\left(\varepsilon_{s}-\bar{\varepsilon}_{p}\right) n_{m}$

where $\bar{a}_{m}$ and $\bar{\varepsilon}_{p}$ are, respectively, the average radius of polymer units and the average permittivity of proteins.

Suppose that the liquid phase is incompressible and has constant physical properties. Then the flow field can be described by the Navier-Stokes equation:

$\eta \frac{d^{2} u}{d x^{2}}+\rho_{e l} E=0, \quad d_{p l} \leq x$

$\eta \frac{d^{2} u}{d x^{2}}-\gamma u+\rho_{e l} E=0, \quad 0 \leq x \leq d_{p l}$

In these expressions, $u$ is the magnitude of the liquid velocity $\boldsymbol{u}$, and $\gamma$ is the friction coefficient per unit volume in the protein layer. According to the pearl bead model for polymer macromolecule hydrodynamics in a dilute solution proposed by Debye [19], $\gamma$ can be expressed as:

$\gamma=6 \pi \eta \bar{a}_{m} n_{m}$

The following boundary conditions are assumed for the electrical field and the flow field:

$$
\begin{aligned}
& \psi \rightarrow 0, \quad \frac{d \psi}{d x} \rightarrow 0, \quad \text { as } x \rightarrow \infty \\
& \left.\psi\right|_{x=d_{p l}^{-}}=\left.\psi\right|_{x=d_{p l}^{+}}, \\
& \left.\left(\varepsilon_{p l} \frac{d \psi}{d x}\right)\right|_{x=d_{p l}^{-}}=\left.\left(\varepsilon_{s} \frac{d \psi}{d x}\right)\right|_{x=d_{p l}^{+}}, \quad x=d_{p l} \\
& \left.\left(\varepsilon_{l l} \frac{d \psi}{d x}\right)\right|_{x=0^{-}}=\left.\left(\varepsilon_{p l} \frac{d \psi}{d x}\right)\right|_{x=0^{+}}=0, \quad x=0 \\
& u \rightarrow U, \quad \frac{d u}{d x} \rightarrow 0, \quad \text { as } x \rightarrow \infty
\end{aligned}
$$

$\left.u\right|_{x=d_{p l}^{-}}=\left.u\right|_{x=d_{p l}^{+}}$,

$\left.\frac{d u}{d x}\right|_{x=d_{p l}^{-}}=\left.\frac{d u}{d x}\right|_{x=d_{p l}^{+}}, \quad x=d_{p l}$

$u=0, \quad x=0$

In these expressions, $\varepsilon_{l l}$ is the permittivity of the lipid bi-layer, and $U$ is the magnitude of liquid velocity far from cell surface $\boldsymbol{U}$, which is defined to be positive in the direction of $\boldsymbol{E}$. Eqs. (9a) and (9d) state that the electrical field and the flow field reduce to their bulk properties at a point far away from cell surface. Eqs. (9b) and (9e) imply that the electric field and the flow field are continuous on the protein layer/liquid interface. The condition specified in Eq. (9c) implies that the lipid bi-layer/ protein layer interface is impermeable to mobile ions, and the electrical filed is absent inside the lipid bi-layer. Expression Eq. (9f) describes the noslip condition for the flow field on the lipid bilayer/protein layer interface.

\section{Results and discussion}

The governing equations of the problem under consideration are solved numerically based on a finite difference method. The electrical potential described by Eqs. (3a) and (3b) is solved first subject to Eqs. (9a), (9b) and (9c), followed by solving the flow field described by Eqs. (7a) and (7b) subject to Eqs. (9d), (9e) and (9f). Once these fields are known, the electrophoretic mobility of a cell can be calculated by:

$\mu_{e}=-\frac{U}{E}$

Table 1 summarizes the relevant properties of erythrocyte reported in the literature [14-16]. In the numerical simulation $\bar{a}_{m}$ is assumed the value of $5 \times 10^{-10} \mathrm{~m}$, which is based on the van der Waals volume of $N$-alkane with similar molecular weight. Fig. 3 illustrates the experimental data for the variation of the electrophoretic mobility of human erythrocyte as a function of $\mathrm{pH}$ reported by Haydon and Seaman [16], and the result predicted by our model. $\mathrm{p} K_{\mathrm{BH}^{+}}$is estimated as 8.5 , which is inferred from the electrophoretic 
Table 1

Relevant data for the electrophoresis of human erythrocyte cell [14-16]

\begin{tabular}{llll}
\hline$T$ & $25{ }^{\circ} \mathrm{C}$ & $C_{\mathrm{HA}_{\text {Naa }} 0}{ }^{0}$ & $0.0288 \mathrm{M}$ \\
$E$ & $200 \mathrm{~V} / \mathrm{m}$ & $C_{\mathrm{HA}_{\alpha-\mathrm{ca}}}$ & $0.0180 \mathrm{M}$ \\
$\eta$ & $1.51 \mathrm{cp}$ & $\mathrm{p} K_{\mathrm{HA}_{\text {Naa }}}$ & 2.6 \\
$\varepsilon_{S}$ & $80.1 \varepsilon_{0}$ & $\mathrm{p} K_{\mathrm{HA}_{\alpha-\mathrm{ca}}}$ & 3.35 \\
$\bar{\varepsilon}_{p}$ & $3.0 \varepsilon_{0}$ & $M_{\mathrm{HA}_{\mathrm{Naa}}}$ & $309 \mathrm{~g} / \mathrm{mol}$ \\
$A_{c m}$ & $1.63 \times 10^{-10} \mathrm{~m}^{2}$ & $\bar{M}_{m}$ & $150 \mathrm{~g} / \mathrm{mol}$ \\
$d_{l l}$ & $75 \times 10^{-10} \mathrm{~m}$ & $\bar{a}_{m}$ & $5 \times 10^{-10} \mathrm{~m}$ \\
$d_{p l}$ & $75 \times 10^{-10} \mathrm{~m}$ & & \\
\hline
\end{tabular}

mobility of cell for $\mathrm{pH}>7$, and $\bar{n}_{m}$ is estimated from the electrophoretic mobility near $\mathrm{pH} 10$, where the dissociation of $\mathrm{BH}^{+}$is almost complete and $C_{\mathrm{BH}^{+}} \cong 0$. Also, $C_{\mathrm{B}}^{0}$ is estimated by fitting the electrophoretic mobility data for the whole range of $\mathrm{pH}$. The estimated values of the relevant parameters are summarized in Table 2. As can be seen from Fig. 3, the performance of the present model is satisfactory. Note that because the dissociation/association of amino base functional groups is not considered in the analysis of Haydon and Seaman [16], their model is not applicable to the case when $\mathrm{pH}$ is higher than 7 . The present model does not have this limitation. Table 2
Table 2

Estimated values of the adjustable parameters used in the theoretical calculations

\begin{tabular}{lll}
\hline $\bar{n}_{m}$ & $3.25 \times 10^{26}$ & \\
& units $/ \mathrm{m}^{3}$ & \\
$\mathrm{p} K_{\mathrm{BH}}$ & 8.5 & \\
$C_{\mathrm{B}}^{0}$ & $0.00105 \mathrm{M}$ & \\
& Present work & $\begin{array}{l}\text { Jamieson and Green- } \\
\text { walt [11] }\end{array}$ \\
& & . \\
\hline
\end{tabular}

Proportionalities $(\%)$ in erythrocyte cell membrane

$\begin{array}{lll}\text { Membrane protein } & 47.83 & 49.20 \\ \text { Membrane lipid } & 44.92 & 43.60 \\ N \text {-acetylneuraminic } & 1.25 & 1.20\end{array}$

acid

reveals that the compositions of erythrocyte membrane estimated by the present model are consistent with those measured experimentally [11].

Fig. 4 illustrates the simulated variation of the electrophoretic mobility of erythrocyte as a function of $\mathrm{pH}$. For comparison, the corresponding electrophoretic mobility for the case when protein units are uniformly distributed in the protein layer is also illustrated in this figure. The spatial variation of the scaled number concentration of protein units, $n_{m} / \bar{n}_{m}$, and that of the scaled

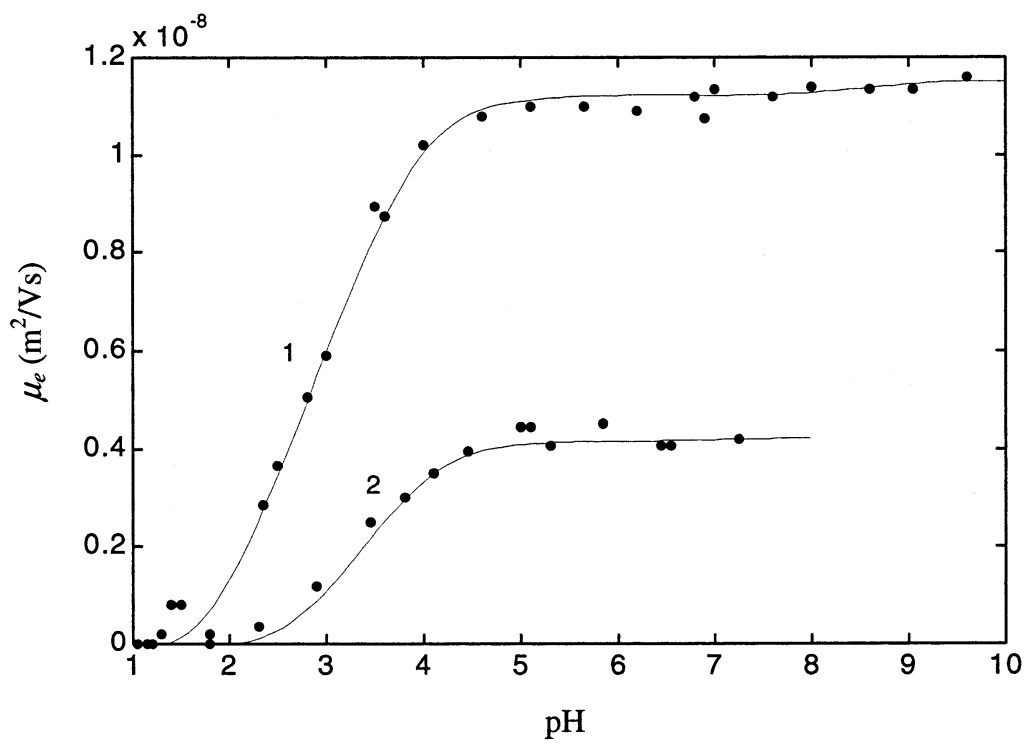

Fig. 3. Variation of electrophoretic mobility $\mu_{e}$ as a function of $\mathrm{pH}$ under various conditions for the case of the concentration of $\mathrm{NaCl}$ $C_{\mathrm{NaCl}}=0.145$ M. 1, treated with acetaldehyde; 2, treated with acetaldehyde and neuraminidase successively. Discrete symbols, experimental data of Haydon and Seaman [16], curves, results based on present model. 


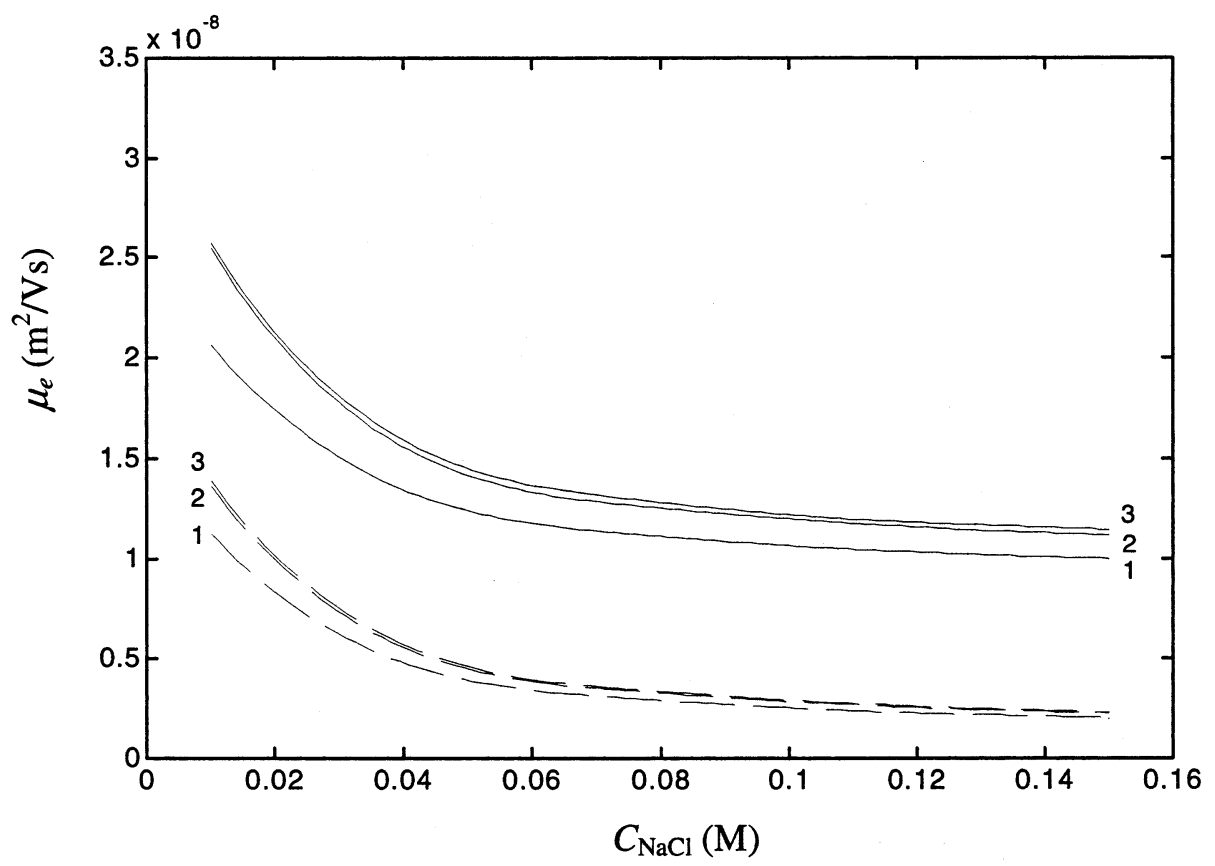

Fig. 4. Variation of the electrophoretic mobility $\mu_{e}$ as a function of $C_{\mathrm{NaCl}}$ at various pH. $1, \mathrm{pH} 4 ; 2, \mathrm{pH} 7 ; 3$, pH 10. Solid curves, present model; dash curves, protein units are uniformly distributed in protein layer.

permeability of protein layer, $\varepsilon_{p l} / \varepsilon_{s}$, are shown in Fig. 5. The spatial variations of electrical potential at three levels of $\mathrm{pH}$ are presented in Fig. 6. Fig. 4 shows that, regardless of the distribution of protein units, the electrophoretic mobility decreases monotonically with the increase in the ionic strength. This behavior is different from that of a rigid entity maintained at either constant

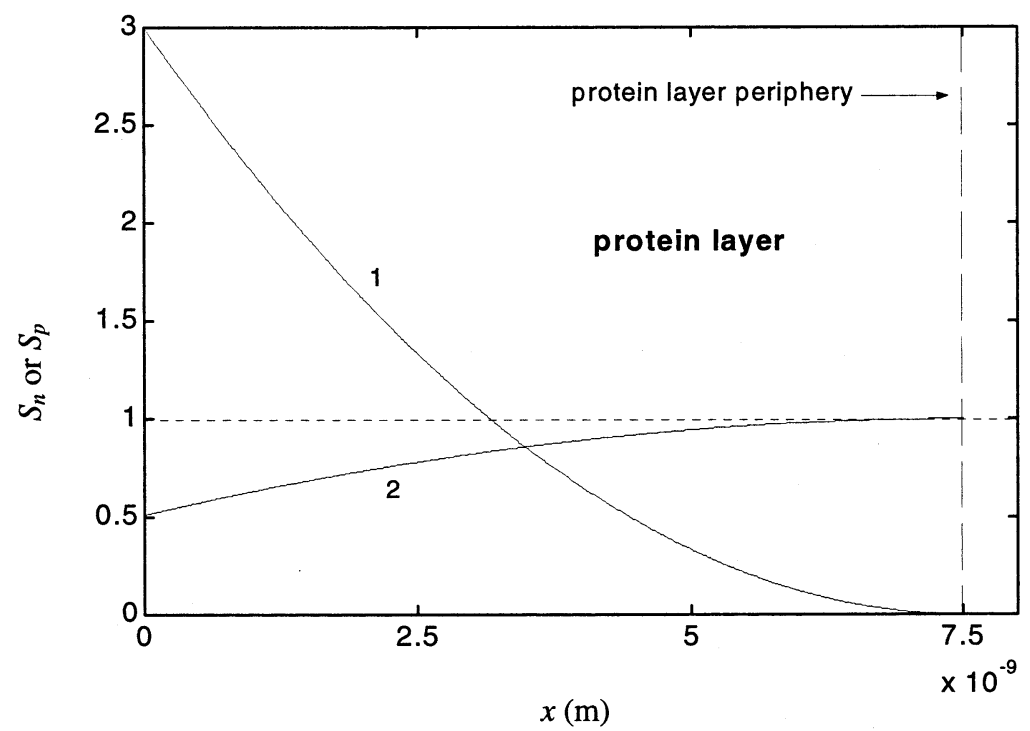

Fig. 5. Spatial variations of the scaled number concentration of protein units in protein layer, $S_{n}=n_{m} / \bar{n}_{m}$, and the scaled permittivity of protein layer, $S_{p}=\varepsilon_{p l} / \varepsilon_{s}$. 1, $S_{n} ; 2, S_{p}$. 


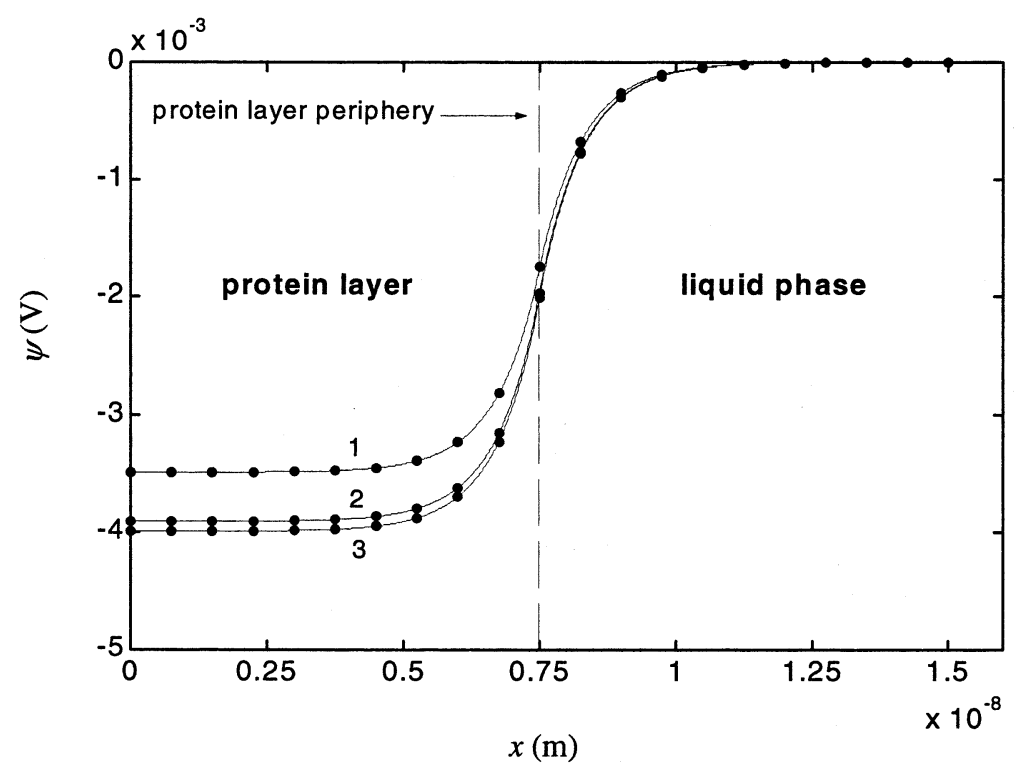

Fig. 6. Spatial variation of electrical potential $\psi$ for various $\mathrm{pH}$ at $C_{\mathrm{NaCl}}=0.15 \mathrm{M} .1, \mathrm{pH} 4 ; 2, \mathrm{pH} 7$; 3 , pH 10. Solid curves, present model; discrete symbols, protein units are uniformly distributed in protein layer.

surface potential or constant surface charge density, where the mobility increases with ionic strength. This is because the higher the ionic strength, the thinner the double layer surrounding an entity, and the greater the gradient of electrical potential near entity surface. In our case, a cell comprises an ion-penetrable, charge-regulated protein layer, and the charged condition inside the layer depends largely on the nature of the surrounding liquid. If double layer is thin, the space available for the distribution of dissociated ionic species inside is limited, so is the degree of dissociation of the functional groups in the protein layer, which leads to a low fixed charge density, and therefore, a small electrophoretic mobility. Fig. 4 reveals that the electrophoretic mobility for the case when protein units are uniformly distributed in the protein layer is smaller than that when they are nonuniformly distributed. This is mainly due to the difference in the frictional force contributed by the protein layer between these two cases. The magnitude of the frictional force is proportional to the relative velocity between the fluid and the protein units. The total frictional force for the case when protein units are nonuniformly distributed in the protein layer is smaller than that when they are uniformly distributed, and therefore, the mobility for the former is lager than that for the latter. Note that although the variation of the permittivity in the protein layer is apparent, as shown in Fig. 5, the effect of the distribution of protein units on the spatial variation of electrical potential is inappreciable, as can be seen in Fig. 6.

Fig. 7 illustrates the typical flow field of the problem under consideration. Both the result for the case when protein units are uniformly distributed in the protein layer and that when they are nonuniformly distributed are presented. Note that inside the protein layer the flow field of the former has a plateau region, which is similar to the result of Levine et al. [4], but the latter does not have this region. The qualitative behavior of the flow field outside the protein layer, however, seems to be unaffected by the distribution of protein units.

Fig. 8 shows the spatial variation of $\mathrm{Na}^{+}$ concentration, scaled by its bulk concentration $C_{\mathrm{Na}^{+}}{ }^{b}$, at various $\mathrm{pH}$. This figure suggests that a higher $\mathrm{pH}$ yield a higher $\mathrm{Na}^{+}$concentration in the protein layer. As pointed out by Michell [20], the balance of $\mathrm{Na}^{+}$between intracellular and extraculluar regions of a cell is attained by influx with 


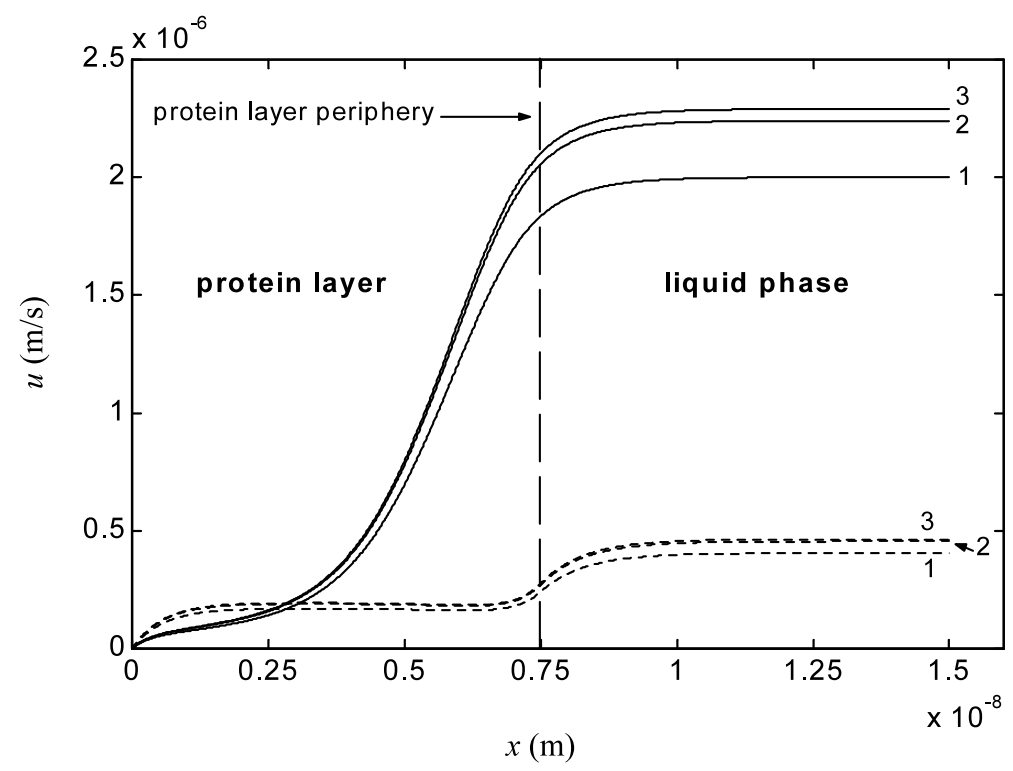

Fig. 7. Spatial variation of liquid velocity $u$ as a function for various $\mathrm{pH}$ at $C_{\mathrm{NaCl}}=0.15$ M. $1, \mathrm{pH} 4 ; 2, \mathrm{pH} 7 ; 3$, $\mathrm{pH} 10$. Solid curves, present model; dash curves, protein units are uniformly distributed in protein layer.

passive transport and outflow with active pumping of cell membrane. The increment of $\mathrm{Na}^{+}$gradient across cell membrane will lead to an accumulation of metabolites for isotonic balance [21], to raise of the concentration of $\mathrm{Na}-\mathrm{K}$ ATPase for gaining more energy from ATP to pump $\mathrm{Na}^{+}$out and $\mathrm{K}^{+}$ in, and to improve the efficiency for the $\mathrm{Na}^{+} / \mathrm{H}^{+}$ exchange inside a cell [20]. The last effect suggests that the variation of $\mathrm{pH}$ in extracellular fluid can have the effect of triggering the self-regulation of

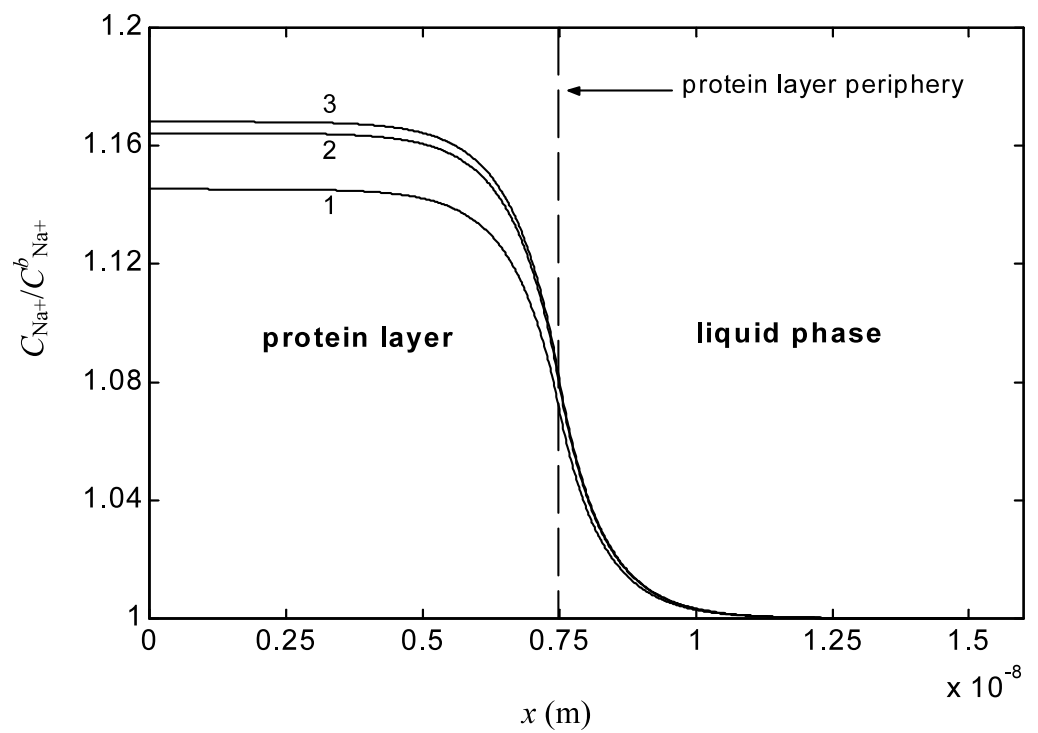

Fig. 8. Spatial variation of $\mathrm{Na}^{+}$concentration, scaled by its bulk concentration $C_{\mathrm{Na}^{+}}{ }^{b}$, at various $\mathrm{pH}$ for the case when $C_{\mathrm{NaCl}}=0.15$ M. Curve 1, pH 4; 2, pH 7; 3, pH 10. 
cell $\mathrm{pH}$. When fresh blood is transported from aorta to blood capillary around tissues, the $\mathrm{pH}$ level of plasma decreases, and the concentration of $\mathrm{Na}^{+}$near cell membrane decreases also, as indicated in Fig. 8. This has the effect of lowering the influx of $\mathrm{Na}^{+}$, and the concentration of $\mathrm{Na}^{+}$ inside a cell is depressed, which is advantageous to the release of cell metabolites to plasma. Also, because the $\mathrm{Na}^{+} / \mathrm{H}^{+}$exchange efficiency inside a cell decreases, the $\mathrm{pH}$ of intracellular fluid decreases accordingly.

\section{Acknowledgements}

This work is supported by the National Science Council of the Republic of China.

\section{References}

[1] R.J. Hunter, Foundations of Colloid Science, vol. 1, Clarendon Press, Oxford, 1992.

[2] I.S. Jones, J. Colloid Interf. Sci. 68 (1979) 451.

[3] R.W. Wunderlich, J. Colloid Interf. Sci. 88 (1982) 385.

[4] S. Levine, M. Levine, K.A. Sharp, D.E. Brooks, Biophys. J. 42 (1983) 27.
[5] H. Ohshima, T. Kondo, J. Colloid Interf. Sci. 116 (1987) 305.

[6] H. Ohshima, M. Nakamura, T. Kondo, Colloid Polym. Sci. 270 (1992) 873.

[7] H. Ohshima, J. Colloid Interf. Sci. 163 (1994) 474.

[8] K. Makino, S. Yamamoto, K. Fujimoto, H. Kawaguchi, H. Ohshima, J. Colloid Interf. Sci. 166 (1994) 251.

[9] J.P. Hsu, S.H. Lin, S. Tseng, J. Theor. Biol. 182 (1996) 137.

[10] S. Tseng, S.H. Lin, J.P. Hsu, Colloids Surf. B 13 (1999) 277.

[11] G.A. Jamieson, T.J. Greenwalt, Red Cell Membrane, J.B. Lippincott, Philadelphia, 1969.

[12] D.H. Heard, G.V.F. Seaman, J. Gen. Physiol. 43 (1960) 635.

[13] G.V.F. Seaman, D.H. Heard, J. Gen. Physiol. 44 (1960) 251.

[14] G.V.F. Seaman, D.H. Heard, Blood 18 (1961) 599.

[15] G.V.F. Seaman, G. Uhlenbruck, Arch. Biochem. Biophys. 100 (1963) 493.

[16] D.A. Haydon, G.V.F. Seaman, Arch. Biochem. Biophys. 122 (1967) 126.

[17] P.S. Vassar, M.J. Kendall, G.V.F. Seaman, Arch. Biochem. Biophys. 135 (1969) 350.

[18] G.V.F. Seaman, P.S. Vassar, M.J. Kendall, Arch. Biochem. Biophys. 135 (1969) 356.

[19] P. Debye, J. Chem. Phys. 14 (1946) 636.

[20] A.R. Michell, The Clinical Biology of Sodium, Pergamon, New York, 1995.

[21] M.A. Mehlman, R.W. Hanson, The Role of Membranes in Metabolic Regulation, Academic Press, New York, 1972. 